\title{
Molecular Characterization and Antimicrobial Susceptibility Patterns of Methicillin-Resistant Staphylococcus aureus Isolates in Tabriz, Northwest of Iran
}

\author{
Mojtaba Nikbakht, ${ }^{1,2,3}$ Mohammad Ahangarzadeh Rezaee,,${ }^{1,2,4,}{ }^{*}$ Alka Hasani, ${ }^{4}$ Mohammad Reza \\ Nahaei, ${ }^{2}$ Javid Sadeghi, ${ }^{2}$ and Sirus Jedari Seifi ${ }^{2}$ \\ ${ }^{1}$ Immunology Research Center, Tabriz University of Medical Sciences, Tabriz, IR Iran \\ ${ }^{2}$ Department of Microbiology, Faculty of Medicine, Tabriz University of Medical Sciences, Tabriz, IR Iran \\ ${ }^{3}$ Student Research Committee, Tabriz University of Medical Sciences, Tabriz, IR Iran \\ ${ }^{4}$ Infectious and Tropical Diseases Research Center, Tabriz University of Medical Sciences, Tabriz, IR Iran \\ "Corresponding author: Mohammad Ahangarzadeh Rezaee, Immunology Research Center, Tabriz University of Medical Sciences, Postal code: 51666-14766, Tabriz, IR Iran. \\ Tel/Fax: +98-4133364661, E-mail: rezaee@tbzmed.ac.ir
}

Received 2016 December 22; Revised 2017 March 22; Accepted 2017 April 04.

\begin{abstract}
Background: Methicillin resistant Staphylococcus aureus (MRSA) are one of the most common and important pathogens, accounting for diverse nosocomial and community-acquired infections. The serious concern about these bacteria is the development of antibiotic resistance.

Objectives: The present study was conducted to investigate the frequency of MRSA strains, their epidemiological and molecular relationships and antibiotic susceptibility patterns of isolated strains from university teaching hospitals of Tabriz, Northwestern Iran (during years 2014 and 2015).

Methods: A total of 215 non-repetitive clinical isolates of S. aureus were identified using standard methods. The MRSA isolates were detected by the combination of phenotypic and genotypic methods. The presence of $p v l$ gene and SCCmec types was determined by PCR and multiplex PCR, respectively. The MRSA isolates, in which the presence of mecA gene had been confirmed by PCR, were subjected to Rep-PCR analysis. Resistance to antibacterial agents was determined by disk diffusion, screening agar, and E-test methods. Results: All S. aureus isolates were positive for nuc gene and 87 (40.5\%) of them revealed the presence of mecA gene, confirming them as MRSA. All isolates were found to be sensitive to linezolid and vancomycin. However, a reduced sensitivity of 3 MRSA isolates to vancomycin was observed (MIC $=6 \mu \mathrm{g} / \mathrm{mL}$ ). SCCmec type III was the most prevalent (79.31\%), followed by type IVd (13.80\%) and type I (6.90\%). The PVL occurrence was detected in 33 (15.35\%) S. aureus isolates. The MRSA isolates could be divided to 2 main clusters, indicating the possible clonal relatedness of MRSA isolates.

Conclusions: The MRSA isolates with SCCmec type III were the predominant MRSA strains in this area. The majority of MRSA isolates were MDR. Linezolid and vancomycin were found as suitable antibiotics for the treatment of MRSA. The results of typing methods indicated possible clonal relatedness among MRSA isolates. Therefore, routine infection control surveillance is necessary for the prevention of epidemic emergence.
\end{abstract}

Keywords: Methicillin-Resistant, Drug-Resistance, Infection Control, Iran, Staphylococcus aureus

\section{Background}

To date, 12 different types of SCCmec (I to XII) have been described for methicillin resistant Staphylococcus aureus (MRSA) isolates (1). Types I-V are globally distributed, yet the others have been limited to geographic locations around the world $(1,2)$. Types I, II, III, VI, and VIII, which are mainly associated with hospital-acquired (HA) infections (HA-MRSA), contain genes that encode resistance to other antimicrobials and are associated with multiple drug resistance (1,3-5). SCCmec types IV and V has commonly been found in community-acquired (CA) methicillin-resistant Staphylococcus aureus (CA-MRSA) strains $(1,5)$. Types IX and
$\mathrm{X}$ are associated with animals (livestock-associated (LA) MRSA or LA-MRSA), of which type IX contains mecC (1).

Panton-valentine leukocidin $(P V L)$ is considered one of the important virulence factors of MRSA and Methicillin Sensitive Staphylococcus aureus (MSSA) isolates responsible for acute coetaneous infections, severe chronic osteomyelitis, deadly necrotizing pneumonia, long-term, and intractable infections $(6,7)$. In the recent years, the prevalence of PVL in S. aureus strains is increasing due to global distribution of PVL-producing MRSA strains. The worldwide spread of PVL-positive CA-MRSA is likely related to continent-specific clones of PVL-positive CA-MRSA, the 
spreading of the isolates from country to country by international travel, and the emergence of new PVL-positive CAMRSA clones with different genetic backgrounds. On the other hand, PVL positive CA-MRSA strains, which were initially susceptible to most anti-staphylococcal antimicrobial agents, have acquired new antimicrobial resistance determinants and thus survive in various environments. Therefore, the combination of the mecA gene and the $p v l$ gene would create a super adaptable $S$. aureus strain, which is multidrug-resistant and capable of rapid spreading around the world, which emphasizes the importance of determining the local prevalence of PVL-positive MRSA strains, as it can be useful in early and proper therapy for serious MRSA infections and their management $(2,5,6,8)$.

Accurate epidemiological typing is of primary importance for the identification of MRSA strains found in hospitals and communities, thereby enabling sources and routes of transmission to be identified and controlled (911).

Repetitive palindromic extragenic elements polymerase chain reaction (Rep-PCR) was described by Versalovic et al. (11,12). The Rep-PCR is an epidemiological typing technique and provides an attractive system, which is discriminative, more rapid, and cost-effective than other molecular typing assays $(11,13,14)$.

The SCCmec typing method has also been used many times to determine outbreaks, epidemiology and lineage of MRSA isolates, recovered from hospitals and communities $(1,5,6)$.

\section{Objectives}

In this study, the frequency of MRSA and its antibiotic resistance pattern, occurrence of the pvl gene, SCCmec types and Rep-PCR typing of MRSA isolates were determined in Tabriz, North West of Iran.

\section{Methods}

\subsection{Isolation and Identification of Staphylococcus aureus}

During a 1-year period, from February, 2014, to March, 2015, all clinical specimens of out-patients and in-patients submitted to microbiology laboratories of educational health care centers of Tabriz University of Medical Sciences, in the Northwest of Iran, were screened for the presence of S. aureus isolates by standard microbiology tests. Repetitive isolates from the same patient were not included in this study.

\subsection{Antibiotic Susceptibility Testing}

Antibiotic susceptibility test was performed as per the clinical and laboratory standards institute (CLSI-2014) guidelines (15), with a panel of the following antibiotics (MAST, Group Ltd, Merseyside, UK): oxacillin ( $1 \mu \mathrm{g})$, cefoxitin $(30 \mu \mathrm{g})$, penicillin (10 $\mathrm{U})$, gentamicin $(10 \mu \mathrm{g})$, erythromycin $(15 \mu \mathrm{g})$, clindamycin $(2 \mu \mathrm{g})$, rifampin (30 $\mu \mathrm{g}$ ), ciprofloxacin (5 $\mu \mathrm{g})$, trimethoprim-sulfamethoxazole (1.25/23.75 $\mu \mathrm{g})$, and linezolid (30 $\mu \mathrm{g})$.

The minimum inhibitory concentrations (MICs) were determined by E-test on Mueller-Hinton's agar plates (MHA, Merck, Germany) for oxacillin and vancomycin, according to the manufacturer's recommendations (Liofilchem, Italy), and the breakpoints for resistance were those defined by the clinical laboratory standards institute (CLSI)-2014.

\subsection{Agar Screening Methods}

Agar screening tests for susceptibility to oxacillin and vancomycin were performed as directed by CLSI-2014 (15).

\subsection{DNA Extraction}

Briefly, DNA was extracted using dodecyl sulfate sodium salt (SDS, Merck, Germany)- proteinase K (CinnaGene, Tehran, Iran) method modified with N-cetyl-N, N, $\mathrm{N}$-trimethyl ammonium bromide (CTAB, Merck, Germany) (2). The concentration of the extracted DNA was confirmed by Nano drop 1000 (NanoDrop, Wilmington, USA).

\subsection{Identification ofnuc, mecA and pvl genes by the Polymerase} Chain Reaction

Confirmation of all S. aureus isolates was carried out by PCR for the amplification of nuc gene (279 bp), as done in the researcher's previous study (16). The PCR reactions were performed in a final volume of $25 \mu \mathrm{L}$ with an automated thermal cycler (Eppendorf mastercycler gradient, Germany) using the primers (CinnaGene, Tehran, Iran) for the nuc gene (Table 1). Negative controls for each primer contained all components except the template DNA.

The $S$. aureus isolates were confirmed as MRSA by the presence of mecA gene ( $310 \mathrm{bp}$ ) among the isolates of $S$. aureus (2).

Amplification of $p v l$ gene (433 bp) among the isolates of $S$. aureus was performed according to a study by Lina et al. (7). Table 1 shows the process and the set of primers used for the amplification of the above-mentioned genes. 
Table 1. Set of Primers Used in this Study

\begin{tabular}{|c|c|c|c|c|}
\hline Primer & Sequence $\left(5^{\prime} \rightarrow 3^{\prime}\right)$ & Amplicon Size, bp & Target Gene & References \\
\hline nuc/F & GCGATTGATGGTGATACGGTT & \multirow{2}{*}{267} & \multirow{2}{*}{ nuc } & \multirow{2}{*}{24} \\
\hline$n u c / \mathbf{R}$ & AGCCAAGCCTTGACGAACTAAAGC & & & \\
\hline mecA/F & GTAGAAATGACTGAACGTCCGATAA & \multirow{2}{*}{310} & \multirow{2}{*}{ mecA } & \multirow{2}{*}{7} \\
\hline $\operatorname{mec} A / \mathrm{R}$ & CCAATTCCACATTGTTTCGGTCTAA & & & \\
\hline$p v l / F$ & ATCATTAGGTAAAATGTCTGGACATGATCCA & \multirow{2}{*}{433} & \multirow{2}{*}{$p v l$} & \multirow{2}{*}{12} \\
\hline$p v l / \mathbf{R}$ & GCATCAASTGTATTGGATAGCAAAAGC & & & \\
\hline$\beta$ & ATTGCCTTGATAATAGCCYTCT & \multirow{2}{*}{937} & \multirow{2}{*}{ SCCmec type II, IV } & \multirow{2}{*}{7.26} \\
\hline $3 \alpha$ & TAAAGGCATCAATGCACAAACACT & & & \\
\hline $\mathbf{c c r C} / \mathbf{F}$ & CGTCTATTACAAGATGTTAAGGATAAT & \multirow{2}{*}{518} & \multirow{2}{*}{ SCCmec type III, V } & \multirow{2}{*}{7.26} \\
\hline $\operatorname{ccrC} / \mathbf{R}$ & CCTTTATAGACTGGATTATTCAAAATAT & & & \\
\hline 1272F1 & GCCACTCATAACATATGGAA & \multirow{2}{*}{415} & \multirow{2}{*}{ SCCmec type I, IV } & \multirow{2}{*}{7.26} \\
\hline 1272R1 & CATCCGAGTGAAACCCAAA & & & \\
\hline 5RmecA & TATACCAAACCCGACAACTAC & \multirow{2}{*}{359} & \multirow{2}{*}{ SCCmec type V } & \multirow{2}{*}{7.26} \\
\hline $5 R 431$ & CGGCTACAGTGATAACATCC & & & \\
\hline IVa-F & GCCTTATTCGAAGAAACCG & \multirow{2}{*}{776} & \multirow{2}{*}{ SCCmec IVa } & \multirow{2}{*}{27} \\
\hline IVa-R & СTACTCTTCTGAAAAGCGTCG & & & \\
\hline IVb-F & TCTGGAATTACTTCAGCTGC & \multirow{2}{*}{493} & \multirow{2}{*}{ SCCmec IVb } & \multirow{2}{*}{27} \\
\hline IVb-R & AAACAATATTGCTCTCCCTC & & & \\
\hline IVc-F & ACAATATTTGTATTATCGGAGAGC & \multirow{2}{*}{200} & \multirow{2}{*}{ SCCmec IVc } & \multirow{2}{*}{27} \\
\hline IVc-R & TTGGTATGAGGTATTGCTGG & & & \\
\hline IVd-F5 & CTCAAAATACGGACCCCAATACA & \multirow{2}{*}{881} & \multirow{2}{*}{ SCCmec IVd } & \multirow{2}{*}{27} \\
\hline IVd-R6 & TGCTCCAGTAATTGCTAAAG & & & \\
\hline (GTG) & GTGGTGGTGGTGGTG & - & Rep & 18 \\
\hline
\end{tabular}

Abbreviations: $F$, forward; $R$, reverse.

\subsection{Methicillin Resistant Staphylococcus aureus Typing}

\subsubsection{Amplification of SCCmec by Multiplex PCR}

Multiplex PCR was performed for the detection of SCCmec types I (415 bp), II (937 bp), III (518 bp), IV (937 bp, 415 $\mathrm{bp}$ ), and V (518 bp, $359 \mathrm{bp}$ ) using 5 sets of primers (Table 1), as previously described $(2,17)$. The multiplex PCR assay for SCCmec IV subtypes was carried out using 4 sets of primers (Table 1), as described previously (18).

3.6.2. Methicillin Resistant Staphylococcus aureus Typing by Rep-Polymerase Chain Reaction

The Rep-PCR was used for the typing of 87 MRSA isolates with the primer of (GTG) 5 (Table 1), as previously described $(13,14)$. The electrophoresis data were analyzed using SPSS 22.0 statistical software (SPSS Inc., Chicago, IL). Dendrogram was built based on Rep-PCR results, using the algidus interval method, on the basis of the centroid profile.

\subsection{Control Strains Used in this Study}

Quality control of discs with antibiotics was performed using S. aureus ATCC 25923, in accordance with the recommendations of CLSI 2014. The presence of nuc gene was controlled by S. aureus ATCC 25923 and S. epidermidis ATCC 12228 as positive and negative control strains, respectively. In the PCR assay for $p v l$ gene, the native isolate of S. aureus with pvl gene and S. aureus ATCC 25923 were used as positive and negative controls, respectively. Methicillin resistance was controlled by standard strains: $S$. aureus ATCC 25923 as the negative control and S. aureus ATCC 33591 as the positive control. For the quality control of vancomycin susceptibility tests, E. faecalis ATCC 29212 and E. faecalis ATCC 51299 were used as positive and negative control strains, respectively. All control isolates of MRSA used 
as the positive control for SCCmec typing were kindly provided by Dr. Mohammad Emaneini (Tehran University of Medical Science, Iran).

\subsection{Statistical Analyses}

Data were analyzed by the chi-square test using the SPSS 22.0 statistical software (SPSS Inc., Chicago, IL). A statistically significant difference was considered as a P value of $<0.05$.

\section{Results}

\subsection{Bacterial Isolation and Confirmation}

A total of 215 isolates of S. aureus were collected from various clinical specimens and identified by standard tests. Table 2 depicts the source of $215 \mathrm{~S}$. aureus isolates. The majority of the isolates were obtained from wound and blood sources.

Table 2. Staphylococcus aureus Isolates and Their Sources, Resistance to Methicillin and Multi-Drug Resistance

\begin{tabular}{lccc}
\hline $\begin{array}{l}\text { Type of } \\
\text { Specimen }\end{array}$ & $\begin{array}{c}\text { No. of S. aureus } \\
\text { Isolates }(\%)\end{array}$ & $\begin{array}{c}\text { No. of MRSA } \\
\text { Isolates (\%) }\end{array}$ & $\begin{array}{c}\text { MDR(\%) in } \\
\text { MRSA Isolates }\end{array}$ \\
\hline Wound & $80(37.2)$ & $40(50)$ & $36(90)$ \\
\hline Blood & $38(16.7)$ & $20(52.63)$ & $19(95)$ \\
\hline Urine & $30(14)$ & $8(26.67)$ & $6(75)$ \\
\hline Abscess & $20(9.3)$ & $4(20)$ & $2(50)$ \\
\hline Sputum & $13(6)$ & $8(61.54)$ & $8(100)$ \\
\hline CSF & $4(1.9)$ & $3(75)$ & $3(100)$ \\
\hline $\begin{array}{l}\text { Other body } \\
\text { fluids }\end{array}$ & $30(14)$ & $4(13.34)$ & $3(75)$ \\
\hline Total & $215(100)$ & $87(40.5)$ & $77(88.50)$ \\
\hline
\end{tabular}

Abbreviation: MRSA, methicillin-resistant Staphylococcus aureus.

${ }^{a}$ Multi-drug resistant (isolates which were resistant to 3 and more antimicrobial agents, with $88.50 \%$ of the MRSA isolates being MDR).

${ }^{\mathrm{b}}$ The rate of MRSA isolates among 215 Staphylococcus aureus isolates was $40.5 \%$.

\subsection{Phenotypic Tests}

\subsubsection{Antimicrobial Resistance}

The results of antimicrobial sensitivity test showed that all isolates were susceptible to linezolid (100\% susceptible) and the majority (96.3\%) was resistant to penicillin. Rates of resistance in MRSA isolates against the studied antibiotics were as follows: penicillin, $100 \%$; oxacillin, $89.7 \%$; cefoxitin, 96.6\%; clindamycin, 88.5\%; erythromycin, 86.2\%; ciprofloxacin, 83.9\%; gentamicin, 83.9\%; trimethoprimsulfamethoxazole, $55.17 \%$; and rifampin, 39.08\%.
Antibiotic resistance patterns of 87 MRSA isolates are shown in Table 3. The isolates fell to 10 distinctive antibiotic resistance profiles (R1 up to R10). There were 2 major profiles consisting of 39 (R3) and 26 (R4) isolates (Table 3). Of the 87 MRSA isolates, 77 isolates (88.50\%) were resistant to more than 3 antimicrobial agents and were recorded as Multidrug-Resistant (MDR) (Tables 2 and 3).

Table 3. Antibiotic Resistance Patterns of 87 Methicillin Resistant Staphylococcus aureus (MRSA) Isolates

\begin{tabular}{|c|c|c|c|}
\hline $\begin{array}{l}\text { Resistance } \\
\text { Patterns }\end{array}$ & $\begin{array}{l}\text { No. (Identity } \\
\text { of Isolates) }\end{array}$ & $\begin{array}{c}\text { Antibiotic } \\
\text { Resistance } \\
\text { Patterns }\end{array}$ & $\begin{array}{l}\text { MDR }^{\mathrm{a}} \text { or } \\
\text { Non-MDR }\end{array}$ \\
\hline $\mathbf{R}_{1}$ & $4(14,75,111,119)$ & P, OX, FOX & Non-MDR \\
\hline $\mathbf{R}_{\mathbf{2}}$ & $2(132,135)$ & $\begin{array}{c}\text { P, OX, FOX, GM, } \\
\text { CC, SXT }\end{array}$ & MDR \\
\hline $\mathbf{R}_{3}$ & $\begin{array}{c}39(2-5,8,15,25, \\
37,41,42,44,47, \\
61-63,91,98,107, \\
114,117,118,123, \\
124,136,140,151, \\
152,154,156-158, \\
160,162,173,175, \\
176-178,209)\end{array}$ & $\begin{array}{c}\text { P, OX, FOX, GM, } \\
\text { CC, E, CP, SXT }\end{array}$ & MDR \\
\hline $\mathbf{R}_{\mathbf{4}}$ & $\begin{array}{c}26(10,11,13,16, \\
19,22,28,40,48, \\
57,76,83,96,97, \\
105,153,155,159, \\
161,174,181,182, \\
201-203,206)\end{array}$ & $\begin{array}{l}\text { P, OX, FOX, GM, } \\
\text { CC, E, CP, R }\end{array}$ & MDR \\
\hline $\mathbf{R}_{5}$ & $\begin{array}{c}6(60,68,74,78 \\
125,179)\end{array}$ & $\begin{array}{l}\text { P, OX, FOX, GM, } \\
\text { CC, E, CP, SXT, R }\end{array}$ & MDR \\
\hline $\mathbf{R}_{6}$ & $1(192)$ & $\begin{array}{c}\text { P, OX, FOX, CC, E, } \\
\text { CP, R }\end{array}$ & MDR \\
\hline $\mathbf{R}_{7}$ & $1(7)$ & $\begin{array}{c}\text { P, FOX, CC, E, CP, } \\
\text { SXT }\end{array}$ & MDR \\
\hline $\mathbf{R}_{8}$ & $2(20,147)$ & P, FOX, CC, E & MDR \\
\hline $\mathbf{R}_{\mathbf{9}}$ & $3(109,115,150)$ & P, FOX & Non-MDR \\
\hline $\mathbf{R}_{10}$ & $3(39,50,51)$ & $\mathrm{P}$ & Non-MDR \\
\hline
\end{tabular}

Abbreviation: CC, clindamycin; CP, ciprofloxacin; FOX, cefoxitin; GM, gentamicin; E, erythromycin; OX, oxacillin; P, penicillin; R, rifampin; SXT, trimethoprim-sulfamethoxazole.

${ }^{a}$ Multidrug-resistant (isolates which were resistant to 3 and more antimicrobial agents, with $88.50 \%$ of the MRSA isolates being MDR).

\subsubsection{Agar Screening Methods}

By using vancomycin agar screening methods, 3 MRSA isolates were grown on vancomycin agar screening plates. Among the 215 S. aureus isolates, 84 were raised on oxacillin agar screening plates.

\subsubsection{Minimum Inhibitory Concentration (by E-Test)}

The MIC of isolates against vancomycin was in the range of $0.25 \mu \mathrm{g} / \mathrm{mL}$ to $6 \mu \mathrm{g} / \mathrm{mL}$ and the $\mathrm{MIC}_{50}$ and $\mathrm{MIC}_{90}$ for the isolates were 0.5 and $1 \mu \mathrm{g} / \mathrm{mL}$, respectively. Only 3 
MRSA isolates showed MICs equal to $6 \mu \mathrm{g} / \mathrm{mL}$, which were previously grown on vancomycin agar screening plates. These isolates did not fall on the vancomycin resistant category, according to CLSI 2014, therefore, they were considered as vancomycin intermediate-resistant S. aureus (VISA).

The MIC of all $S$. aureus isolates against oxacillin was in the range of $0.25 \mu \mathrm{g} / \mathrm{mL}$ to $256 \mu \mathrm{g} / \mathrm{mL}$. The majority of MRSA isolates were found to be highly resistant(MIC $\geq 256$ $\mathrm{mg} / \mathrm{L})$ to oxacillin $\left(\mathrm{MIC}_{50}=256 \mu \mathrm{g} / \mathrm{mL} ; \mathrm{MIC}_{90}=256 \mu \mathrm{g} / \mathrm{mL}\right)$.

\subsection{Methicillin Resistant Staphylococcus aureus Detection}

By the combination of genotypic (amplification of mecA gene) and phenotypic tests (oxacillin and cefoxitin disk agar diffusion, oxacillin screening agar, and E-test for oxacillin), 87 (40.5\%) isolates were found as MRSA and 128 (59.5\%) as MSSA.

There were absolute correlations between the results of agar screening plates, cefoxitin disk, and E-test in detection of MRSA isolates. By using the above phenotypic methods, and amplification of mecA gene, 84 and 87 isolates were found as MRSA, respectively. Therefore, 3 mecApositive isolates (pre-MRSA or cryptic isolates) could not be distinguished from the mecA negative isolates by the phenotypic tests. The frequency of MRSA isolates is shown in Table 2 based on the sources of isolation.

\subsection{Molecular Tests}

\subsubsection{Identification of nuc, mecA and pvl Genes}

The nuc gene ( $279 \mathrm{bp}$ ) was amplified by all of the isolates. When PCR for mecA gene was performed on S. aureus isolates, 87 (40.5\%) were found as MRSA and 128 (59.5\%) as MSSA.

Of 215 S. aureus isolates, the $p v l$ gene (433 bp fragment) was detected in 33 (15.35\%) of the isolates. Among these, 9 (10.34\%) isolates were MRSA and 24 (18.75\%) were MSSA ( $\mathrm{P}=$ $0.093)$.

\subsubsection{Methicillin Resistant Staphylococcus aureus Typing 4.4.2.1. SCCmec Typing}

All isolates were typeable with the predominance of SCCmec type III, which was detected in 69 (79.31\%) of the isolates followed by type IV $(12,13.80 \%)$ and type I $(6,6.90 \%)$ (Figure 1). All 12 MRSA isolates with SCCmec type IV were subtyped as SCCmec type IVd.

\subsubsection{Rep-Polymerase Chain Reaction}

By using the Rep-PCR assay, 11 different patterns were obtained from 87 MRSA isolates with $100 \%$ similarity (Figure 2). There were 2 major clusters consisting of 58 and 17 isolates (indicating their molecular clonality).
Figure 1. SCCmec patterns of Methicillin Resistant Staphylococcus aureus Isolates by Multiplex Polymerase Chain Reaction

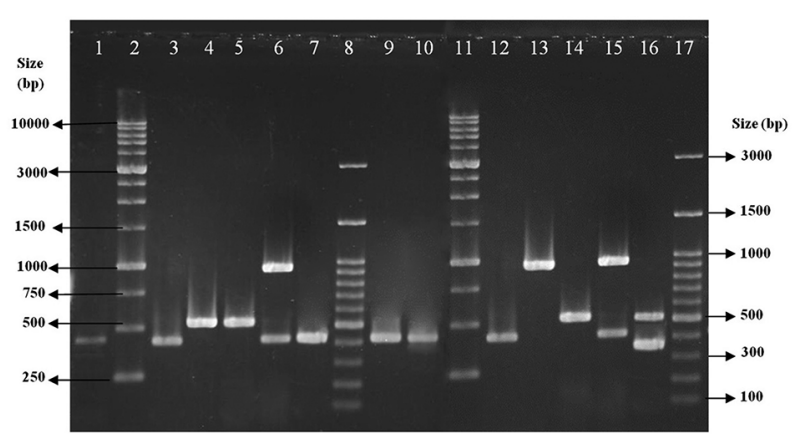

Lanes 1, 3, 7, 9 and 10, clinical isolates with SCCmec type I; lanes 2 and 11, size markers ( 1 kb DNA Ladder); lanes 4 and 5, clinical isolates with SCCmec type III; lane 6, clinical isolate with SCCmec type IV; lanes 8 and 17, size markers (100 bp DNA ladder Plus); and lanes $12-16$, control strains (types I-V).

\section{Discussion}

This study was conducted to investigate antibiotic susceptibility pattern, frequency, and molecular characterization of MRSA isolates in northwest of Iran. The clonal relatedness of isolates was also studied.

In the current study, the frequency of MRSA was $40.50 \%$, which was slightly higher than that reported by similar reports from Iran (36\%), similar to that reported by Fatholahzadeh et al. and a previous study by the current researchers conducted during 2005 and 2012 (35.7\%) in the Northwest of Iran $(16,19)$. However, a higher rate of MRSA was reported by Sadeghi et al. (56.79\%) from the Southeast of Iran (2). In 2 studies performed in Turkey, the rate of MRSA was reported as $24.8 \%$ and $50.2 \%(20,21)$. In some European countries, the frequency of MRSA ranged from $0.6 \%$ to $45 \%$ and in various locations of Russia, it was between $0 \%$ and $89.5 \%$ (20).

Therefore, MRSA rates varied greatly among different countries, probably reflecting differences in infection control activities, time of the study, number of cases studied, and biological characteristics of the MRSA strains (19, 20, $22)$. On the other hand, this variation in the prevalence of MRSA around the world emphasized the importance of determining the local prevalence of MRSA, as it could be useful in empiric therapy and its management $(19,20,22)$.

In the present study, all isolates were found susceptible to vancomycin and linezolid, which is compatible with other published reports $(23,24)$. This may be due to the decrease in the selection pressure for vancomycin antibiotic and no prescription of linezolid in the studied region.

Oxacillin MIC was more than $256 \mu \mathrm{g} / \mathrm{mL}$ for the majority of MRSA isolates $\left(\mathrm{MIC}_{50}=256 \mu \mathrm{g} / \mathrm{mL} ; \mathrm{MIC}_{90}=256\right.$ 


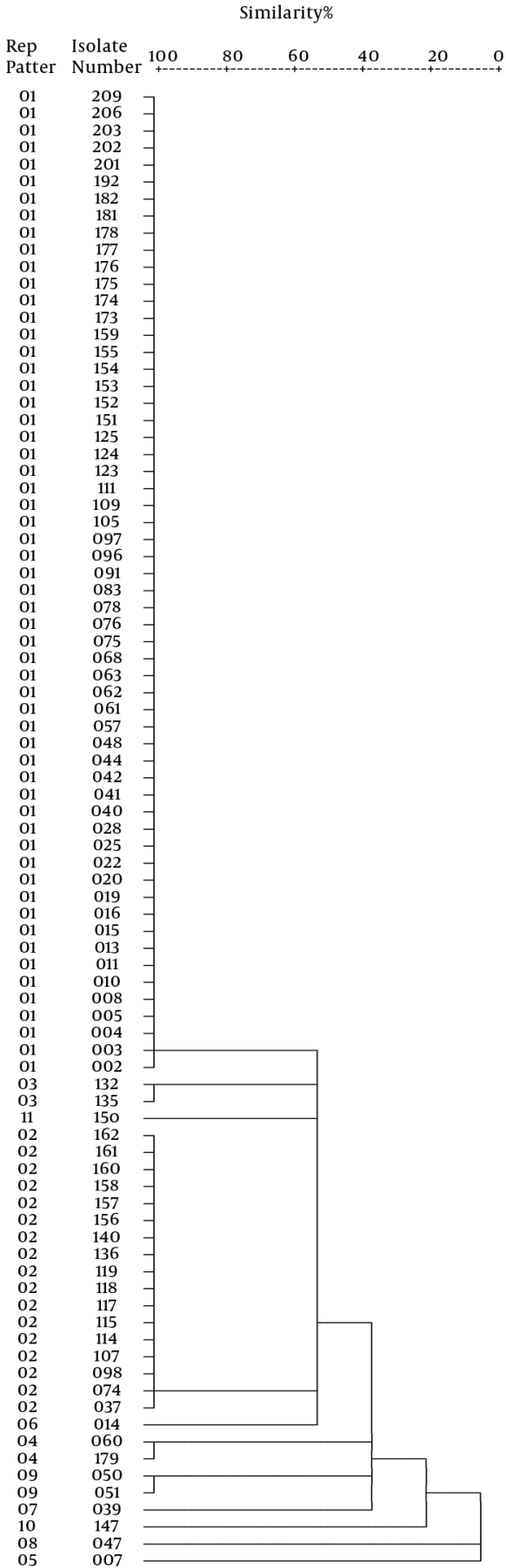

Figure 2. Dendrogram Showing Similarities Between 87 Isolates of Methicillin Resistant Staphylococcus aureus Based on Rep-Polymerase Chain Reaction Data

$\mu \mathrm{g} / \mathrm{mL}$ ), thus indicating that the corresponding genes were efficiently expressed $(19,22)$. These findings could be due to the irrational antibiotic usage in this region for treatment and prophylactic purposes.

The SCCmec typing revealed that SCCmec type III was predominant (69 isolates, 79.31\%) among our 87 MRSA isolates, followed by type IVd $(12,13.80 \%)$ and type I $(6,6.90 \%)$. The results of this study were in agreement with the author's previous findings, showing the predominance of SCCmec III (69.8\%) during 2005 to 2012 in the Northwest of Iran (25). In contrast, in 2 studies conducted by Moghadami et al. and Zeinali et al. in Iran, the SCCmec types I and II were found to be more prevalent, respectively $(4,26)$. The predominance of type III in the current study could be because MRSA isolates descended from a common origin, indicating the presence of clonality among the MRSA isolates $(2,4,22)$.

Therefore, in the areas with inappropriate and high rate of antibiotic use, such as healthcare facilities, MRSA strains have been shown to gain diverse types of SCCmec elements and this variation emphasizes the importance of determining local prevalence of SCCmec type, which could be useful in the management of patients $(2,6)$.

Based on the current data, the majority of MRSA isolates carrying SCCmec type III (66, 96\%) showed an MDR phenotype. In the current study, SCCmec typing was correlated well with major antimicrobial susceptibility patterns (R3 and R4).

Panton-valentine Leukocidin is an important factor associated with severe infections and the occurrence of $P V L$ in $S$. aureus strains is increasing due to the global distribution of PVL-producing strains. The worldwide spread of PVL-positive MRSA and variation in the prevalence of these strains around the world emphasizes the importance of determining the local prevalence of PVL-positive MRSA, as it could be useful in empiric therapy and its management $(2,5-7)$. In the current study, 33 (15.35\%) S. aureus isolates were PVL-positive. In a study from Iran, a low frequency of S. aureus isolates (3.08\%) were PVL-positive (2), while in another research, a high number of isolates $(24.20 \%)$ were reported as PVL-positive (8). The high rate of PVL-positive isolates in the present study is an alarm to clinicians, encouraging proper therapy in this region.

The emergence of vancomycin intermediate-resistant S. aureus is a great concern and alarm to clinicians in finding an alternative treatment.

A total of 11 Rep patterns, including 2 main clusters were obtained from 87 MRSA isolates that were $100 \%$ similar, indicating their molecular clonality and shared origins. Therefore, routine infection controls surveillance could be necessary for the prevention of epidemic emergence. The current findings were in agreement with the results of some previous studies $(2,11,14)$.

According to analyzed data, Rep-PCR patterns [pres- 
ence of 2 major cluster, (Figure 2)] correlated well with SCCmec typing results (predominantly those carrying SCCmec type III) and antimicrobial susceptibility profiles (major antimicrobial susceptibility patterns were R3 and R4), confirming the presence of clonality in the studied MRSA isolates.

\subsection{Conclusions}

The current findings showed that the frequency of MRSA isolates was relatively high in the North West of Iran; those carrying the SCCmec type III were multidrugresistant. Linezolid and vancomycin could be suitable antibiotics in the treatment of infections caused by these multi-resistant pathogens. There was good correlation between the results of Rep analysis, SCCmec typing and antibiotic resistance, indicating a common origin and clonality among the MRSA isolates. Therefore, routine infection control surveillance could be necessary for the prevention of epidemic emergence.

\section{Acknowledgments}

The authors would like to appreciate the contribution of the microbiology laboratory personnel of the hospitals of Tabriz University of Medical Sciences for the collection of clinical isolates.

\section{Footnotes}

Authors' Contribution: Mojtaba Nikbakht and Mohammad Ahangarzadeh Rezaee participated in proposing the idea of the study, designing and providing administrative, technical and material support. Mojtaba Nikbakht, Mohammad Ahangarzadeh Rezaee and Javid Sadegi participated in the collection of data and statistical analysis. Mojtaba Nikbakht, Mohammad Ahangarzadeh Rezaee, Alka Hasani and Mohammad Reza Nahaei participated in drafting and critical revision of the manuscript for important intellectual content. Mojtaba Nikbakht, Mohammad Ahangarzadeh Rezaee, Alka Hasani, Mohammad Reza Nahaei and Javid Sadegi participated in the analysis and interpretation of data. Mojtaba Nikbakht carried out all experiments, including phenotypic and molecular experiments. All authors read and approved the final manuscript.

Conflict of Interest: The authors declared that they had no financial and non-financial conflicts of interest.

Funding/Support: This work was supported financially by the immunology research center, Tabriz University of Medical Sciences (grant No. 93-36); it originated from a database prepared for the PhD thesis of the first author, as registered at Tabriz University of Medical Sciences.
Ethical Approval: This work was approved by the ethical committee of Tabriz University of Medical Sciences (reference No. 5/4/3978).

\section{References}

1. Serafini K, Malin-Mayor B, Nich C, Hunkele K, Carroll KM. Psychometric properties of the Positive and Negative Affect Schedule (PANAS) in a heterogeneous sample of substance users. Am J Drug Alcohol Abuse. 2016;42(2):203-12. doi: 10.3109/00952990.2015.1133632. [PubMed: 26905228].

2. Sadeghi J, Mansouri S. Molecular characterization and antibiotic resistance of clinical isolates of methicillin-resistant Staphylococcus aureus obtained from Southeast of Iran (Kerman). APMIS. 2014;122(5):405-11. doi: 10.1111/apm.12158. [PubMed: 24033803].

3. Chambers HF. Methicillin resistance in staphylococci: molecular and biochemical basis and clinical implications. Clin Microbiol Rev. 1997;10(4):781-91. [PubMed: 9336672].

4. Moghadami M, Japoni A, Karimi A, Mardani M. Comparison of community and healthcare-associated MRSA in Iran. Arch Clin Infect Dis. 2010;5(4):206-12.

5. Rybak MJ, LaPlante KL. Community-associated methicillin-resistant Staphylococcus aureus: a review. Pharmacotherapy. 2005;25(1):74-85. doi: 10.1592/phco.25.1.74.55620. [PubMed: 15767223].

6. David MZ, Daum RS. Community-associated methicillin-resistant Staphylococcus aureus: epidemiology and clinical consequences of an emerging epidemic. Clin Microbiol Rev. 2010;23(3):616-87. doi: 10.1128/CMR.00081-09. [PubMed: 20610826]

7. Lina G, Piemont Y, Godail-Gamot F, Bes M, Peter MO, Gauduchon V, et al. Involvement of Panton-Valentine leukocidin-producing Staphylococcus aureus in primary skin infections and pneumonia. Clin Infect Dis. 1999;29(5):1128-32. doi:10.1086/313461. [PubMed:10524952].

8. Havaei S, Moghadam SO, Pourmand M, Faghri J. Prevalence of Genes Encoding Bi-Component Leukocidins among Clinical Isolates of Methicillin Resistant Staphylococcus aureus. Iran J Public Health. 2010;39(1):8-14. [PubMed: 23112984].

9. Nikbakht M, Nahaei MR, Akhi MT, Asgharzadeh M, Nikvash S. Molecular fingerprinting of meticillin-resistant Staphylococcus aureus strains isolated from patients and staff of two Iranian hospitals. J Hosp Infect. 2008;69(1):46-55. doi: 10.1016/j.jhin.2008.01.034. [PubMed: 18358563].

10. Szabo J. Molecular Methods in Epidemiology of Methicillin Resistant Staphylococcus aureus (MRSA): Advantages, Disadvantages of Different Techniques. J Med Microbiol Diagnos. 2014;03(03) doi:10.4172/21610703.1000147.

11. Trindade PA, McCulloch JA, Oliveira GA, Mamizuka EM. Molecular techniques for MRSA typing: current issues and perspectives. Braz J Infect Dis. 2003;7(1):32-43. [PubMed:12807690].

12. Versalovic J, Koeuth T, Lupski JR. Distribution of repetitive DNA sequences in eubacteria and application to fingerprinting of bacterial genomes. Nucleic Acids Res. 1991;19(24):6823-31. [PubMed: 1762913].

13. Manga I, Vyletelova M. Rep-PCR-based typing as a tool for tracking of MRSA infection origin. Acta Universitatis Agriculturae et Silviculturae Mendelianae Brunensis. 2013;60(6):251-6. doi: 10.11118/actaun201260060251.

14. Svec P, Pantucek R, Petras P, Sedlacek I, Novakova D. Identification of Staphylococcus spp. using (GTG)(5)-PCR fingerprinting. Syst Appl Microbiol. 2010;33(8):451-6. doi: 10.1016/j.syapm.2010.09.004. [PubMed: 21095086].

15. Clinical and Laboratory Standard Institute (CLSI) . Performance Standard for Antimicrobial Susceptibility Testing. M100-S24. CLSI document; 2014 
16. Ahangarzadeh Rezaee M, Mirkarimi SF, Hasani A, Sheikhalizadeh V, Soroush MH, Abdinia B. Molecular Typing of Staphylococcus aureus Isolated From Clinical Specimens During an Eight-Year Period (2005 - 2012) in Tabriz, Iran. Archives of Pediatric Infectious Diseases. 2016;4(2):e35563. doi: 10.5812/pedinfect.35563.

17. Boye K, Bartels MD, Andersen IS, Moller JA, Westh H. A new multiplex PCR for easy screening of methicillin-resistant Staphylococcus aureus SCCmec types I-V. Clin Microbiol Infect. 2007;13(7):725-7. doi: 10.1111/j.1469-0691.2007.01720.x. [PubMed: 17403127].

18. Zhang K, McClure JA, Elsayed S, Louie T, Conly JM. Novel multiplex PCR assay for characterization and concomitant subtyping of staphylococcal cassette chromosome mec types I to V in methicillin-resistant Staphylococcus aureus. J Clin Microbiol. 2005;43(10):5026-33. doi: 10.1128/JCM.43.10.5026-5033.2005. [PubMed:16207957].

19. Fatholahzadeh B, Emaneini M, Gilbert G, Udo E, Aligholi M, Modarressi $\mathrm{MH}$, et al. Staphylococcal cassette chromosome mec (SCCmec) analysis and antimicrobial susceptibility patterns of methicillinresistant Staphylococcus aureus (MRSA) isolates in Tehran, Iran. Microb Drug Resist. 2008;14(3):217-20. doi: 10.1089/mdr.2008.0822. [PubMed: 18694326].

20. Eksi F, Gayyurhan ED, Bayram A, Karsligil T. Determination of antimicrobial susceptibility patterns and inducible clindamycin resistance in Staphylococcus aureus strains recovered from southeastern Turkey. J Microbiol Immunol Infect. 2011;44(1):57-62. doi: 10.1016/j.jmii.2011.01.011. [PubMed: 21531354].

21. Yilmaz G, Aydin K, Iskender S, Caylan R, Koksal I. Detection and prevalence of inducible clindamycin resistance in staphylococci. J Med Microbiol. 2007;56(Pt 3):342-5. doi: 10.1099/jmm.0.46761-0. [PubMed: 17314364].

22. Japoni A, Jamalidoust M, Farshad S, Ziyaeyan M, Alborzi A, Japoni S, et al. Characterization of SCCmec types and antibacterial susceptibility patterns of methicillin-resistant Staphylococcus aureus in Southern Iran.Jpn J Infect Dis. 2011;64(1):28-33. [PubMed: 21266752].

23. Dibah S, Arzanlou M, Jannati E, Shapouri R. Prevalence and antimicrobial resistance pattern of methicillin resistant Staphylococcus aureus (MRSA) strains isolated from clinical specimens in Ardabil, Iran. Iran J Microbiol. 2014;6(3):163-8. [PubMed: 25870749].

24. Hasani A, Sheikhalizadeh V, Hasani A, Naghili B, Valizadeh V, Nikoonijad AR. Methicillin resistant and susceptible Staphylococcus aureus: Appraising therapeutic approaches in the Northwest of Iran. Iran JMicrobiol. 2013;5(1):56-62. [PubMed: 23467268].

25. Fateh Amirkhiz M, Ahangarzadeh Rezaee M, Hasani A, Aghazadeh M Naghili B. SCCmec Typing of Methicillin-Resistant Staphylococcus aureus: An Eight Year Experience. Arch Pediatr Infect Dis. 2015;3(4) doi: 10.5812/pedinfect.30632.

26. Zeinali E, Moniri R, Safari M, Mousavi SGA. Molecular characterization and SCCmec typing in meticillin-resistant Staphylococcus aureus isolated from clinical samples. KAUMS Journal (FEYZ). 2011;14(4):439-46. 